\title{
Alfvén waves damping in protostellar disks providing a way to expand the action of the magneto-rotational instability
}

\author{
A. A. Vidotto and V. Jatenco-Pereira ${ }^{1}$ \\ ${ }^{1}$ IAG, Univ. de São Paulo, Rua do Matão 1226, São Paulo, SP 05508-900, Brazil \\ email: aline@astro.iag.usp.br
}

\begin{abstract}
In order for the magneto-rotational instability to take place, we need a sufficiently ionized disk. Here, we study, besides viscous dissipation, another heating mechanism for the disk that involves the damping of Alfvén waves due to its interaction with dust grains.
\end{abstract}

Keywords. stars: pre-main-sequence, accretion, accretion disks, MHD, turbulence, waves

The most accepted mechanism for redistributing angular momentum in an accretion disk is the magneto-rotational instability. This mechanism only exists if the gas in the disk is sufficiently ionized to be coupled to the magnetic field lines. Hence, a heating mechanism for the disk is needed. Besides the viscous heating mechanism often included in the models by means of the $\alpha$ prescription (Shakura \& Sunyaev 1973), in this work we study the damping of Alfvén waves as an additional heating source. The mechanism we suggest for the damping of the waves involves dust grains, usually observed in these systems. This damping affects the Alfvén wave propagation near the dust-cyclotron frequency, since charged grains in a magnetized medium are highly coupled to the waves due to cyclotron resonances (for further details, see Vidotto \& Jatenco-Pereira 2006). We assume a size grains distribution that implies different charges and thus a broad band of resonance frequencies are damped.

By assuming an initial energy flux of Alfvén waves, $\phi=v_{A}\left\langle(\delta B)^{2}\right\rangle /(4 \pi)$, where $v_{A}$ is the Alfvén velocity and $\delta B$ is the magnetic field perturbation, we analyse how the damping of the waves can increase the disk midplane temperature $T_{c}$. Writing $|\delta B|=f B$, we study the profile of $T_{c}$ for the first $2 \mathrm{AU}$ from the central star for different values of $f(f=0$ means that the only heating mechanism is viscous dissipation). As a result we obtain that the temperature increases more significantly for the outer radii. Comparing with the temperature due to the viscous heating mechanism, if $f=1 \%$, at 0.1 AU there is not any appreciable increase in $T_{c}$ and at $2 \mathrm{AU}$ the increase is $\sim 9 \%$. For $f=10 \%$, this scenario changes: at $0.1 \mathrm{AU}$ the increase in $T_{c}$ is $\sim 10 \%$ and at $2 \mathrm{AU}$ the temperature increases more than 2.5 times. These results show that the wave damping can be an important mechanism for disk heating.

\section{Acknowledgements}

This work is supported by FAPESP (04/13846-6) and CNPq (303459/2004-2).

\section{References}

Shakura, N.I. \& Sunyaev, R.A. 1973, A\&A 24, 337

Vidotto, A.A. \& Jatenco-Pereira, V. 2006, ApJ 639, 416 